\title{
Les peptides antibactériens inductibles des insectes
}

\section{Jules A. Hoffmann Jean-Luc Dimarcq Philippe Bulet}

\section{Remerciements}

Les auteurs tiennent à remercier leurs collègues Danièle Hoffmann, Charles Hétru, Jean-Marc Reichhart, Marie Lagueux et Marie Meister pour discussions et lecture du manuscrit. Ils expriment leur recon naissance au Pr Marius Ptak (centre de biophysique du Cnrs, Orléans) et à ses collaborateurs pour les données sur la structure tridimensionnelle de la défensine A, et au Dr Geoff Richards (laboratoire de génétique moléculaire des eucaryotes, Strasbourg) pour sa collaboration dans l'établissement de lignées transgéniques de drosophile.

\section{ADRESSE}

J. A. Hoffmann : directeur de recherche. J. L. Dimarcq : chargé de recherche. P. Bulet chargé de recherche. Laboratoire de biologie générale de l'université Louis-Pasteur et unité de recherche associée au Cnrs $\mathrm{n}^{\circ} 1490$, Bases moléculaires de la réponse immunitaire des insectes, 12 , rue de l'Uni-

Les insectes supérieurs combattent les agressions bactériennes par un mécanisme très efficace qui comporte la synthèse de plusieurs familles de peptides antibactériens à large spectre d'activité. Outre les cécropines et les défensines d'insectes, les séquences peptidiques de sept autres familles de peptides antibactériens inductibles sont aujourd'hui connues. La synthèse de ces molécules a lieu dans le corps gras, un équivalent fonctionnel du foie des vertébrés. La réponse antibactérienne est activée via des récepteurs à distribution non clonale qui reconnaissent des motifs structuraux largement distribués dans les enveloppes des procaryotes. C'est un phénomène rapide, limité dans le temps et qui, par de nombreux aspects, s'apparente à la réponse de phase aiguë des mammifères. Bien que très active, cette forme primitive de réponse immunitaire ne comporte pas de mémoire.

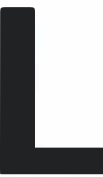

es insectes forment une classe zoologique dont le rayonnement a été remarquable au cours de l'évolution : près de $90 \%$ des espèces animales actuellement connues appartiennent à ce groupe qui occupe pratiquement toutes les niches écologiques sur tous les continents. Un des éléments qui ont certainement contribué au succès évolutif des insectes est l'efficacité de leur défense antibactérienne. Celle-ci avait déjà frappé les auteurs au siècle passé, et Cuénot avait fait, dans les années 1890 à Nancy, de remarquables études sur la phagocytose des bactéries chez le grillon [1]. Un pas important dans la compréhension de la réponse antibactérienne des insectes fut franchi, après la Première Guerre mondiale, grâce aux études indépendantes menées à la station séricicole de Saint-Genis-Laval (Lyon) par Paillot [2] et à l'Institut Pasteur de Paris par un groupe de chercheurs russes originaires de SaintPétersbourg, animé par Metalnikow [3]. Ces travaux établirent que l'injection de doses faibles de bacté- ries à des chenilles (Galleria mellonella) induit en quelques heures l'apparition d'une protection efficace contre l'inoculation subséquente de doses normalement mortelles. Pendant le demisiècle qui a suivi, ces études furent confirmées et étendues à de nombreuses espèces d'insectes appartenant à des ordres divers mais, à part la mise en évidence du lysozyme ubiquitaire [4], les molécules responsables de la protection induite restaient inconnues. Ce n'est qu'au début des années 1980 que Boman et al. (institut de microbiologie de l'université de Stockholm, Suède) réussirent la première caractérisation d'une substance antibactérienne induite dans la pupe diapausante d'un lépidoptère, Hyalophora cecropia [5]. Il s'agissait d'un peptide cationique de petite taille, puissamment actif contre les germes à Gram négatif et positif, qui fut appelé cécropine (de cecropia). Celleci, dont on connaît maintenant plusieurs isoformes, n'est cependant qu'un peptide antibactérien inductible parmi de nombreux autres, et la liste des molécules caractérisées chez 
les insectes s'allonge rapidement. Pour l'instant, les travaux ont porté sur les ordres phylogénétiquement récents (insectes dits supérieurs) : il s'agit des lépidoptères, diptères, hyménoptères et coléoptères.

Tout en soulignant que nos connaissances sur la réponse antibactérienne humorale (par opposition à la réponse cellulaire dont les éléments prédominants sont la phagocytose et l'encapsulation) sont encore très fragmentaires, nous pouvons dégager deux grands traits qui la caractérisent chez tous les insectes étudiés. En premier lieu, il s'agit d'un phénomène inductible qui se traduit par l'apparition dans le sang d'une forte activité antibactérienne. Les inducteurs peuvent être très variés : bactéries, lipopolysaccharides, peptidoglycanes, blessure tégumentaire, etc. La réponse est bien apparente quelques heures après l'injection de bactéries et persiste plusieurs jours. En deuxième lieu, la réponse antibactérienne implique la synthèse chez le même individu de plusieurs molécules antibactériennes de nature peptidique (ou de plusieurs familles de molécules) dont les spectres d'activité sont en général assez larges. A titre d'exemple, nos données actuelles sur la réponse antibactérienne de la drosophile montrent que celle-ci synthétise au moins une dizaine de peptides antibactériens différents dans les heures qui suivent

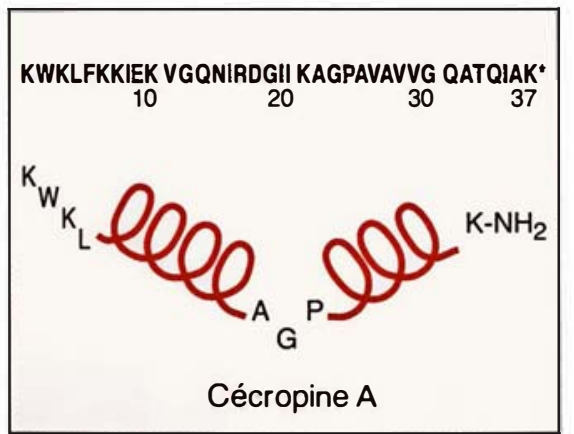

Figure 1. La cécropine A du lépidoptère Hyalophora cecropia : séquence peptidique (code à une lettre) et représentation tridimensionnelle indiquant la présence de deux hélices $\alpha$ séparées par un coude. A noter que les quatre premiers résidus $\mathrm{N}$-terminaux ne sont pas inclus dans l'hélice proximale et que la cécropine est amidée au niveau $C$ terminal. (D'après $[5,6,10]$; représentation tridimentionnelle inspirée de [6].)

$\mathrm{m} / \mathrm{s} n^{\circ} 5$, vol. 8, mai 92 une blessure septique. Pendant le même temps, plusieurs dizaines d'autres protéines sont synthétisées de novo ou voient leur taux de synthèse accru : il s'agit notamment de protéines de reconnaissance et de signalisation.

Pour être complet, il faudrait ajouter à cette liste, le lysozyme qui est présent chez de nombreux insectes, à la fois dans le sang et le tube digestif. Sa présence peut être constitutive ou inductible. Nous ne traiterons pas de cette molécule dans cette revue.

Nous présenterons ci-dessous les données actuelles sur la structure, la biosynthèse et le mode d'action des peptides antibactériens inductibles. Nous analyserons également le contrôle de l'expression de leurs gènes et la reconnaissance de l'agression bactérienne.

\section{Aspects structuraux}

Les informations structurales les plus complètes concernent actuellement deux familles de peptides antibactériens inductibles d'insectes : les cécropines, qui sont dépourvues de cystéines, et les défensines d'insectes, qui ont trois ponts disulfure et présentent une certaine similitude de séquence avec les défensines des mammifères. Nous possédons la séquence de sept autres familles de peptides antibactériens inductibles, mais ne disposons que d'informations réduites sur leur structure et leur mode d'action.

\section{- Les cécropines}

Comme indiqué plus haut, au début des années 1980, le groupe de Boman réussit la purification à partir du sang "immun " (c'est-à-dire prélevé quelques heures après inoculation d'une faible dose de bactéries) de pupes diapausantes du lépidoptère Hyalophora cecropia, de petits peptides cationiques capables de lyser $E$. coli. Le séquençage de ces peptides montrait qu'il s'agissait de molécules nouvelles auxquelles fut donné le nom de cécropines [5]. Actuellement, une dizaine de cécropines ont été isolées et caractérisées chez plusieurs espèces d'insectes [6], qui cependant appartiennent toutes à deux ordres récents : les lépidoptères et les diptères. Il n'est pas certain pour l'instant que ces peptides antibactériens soient largement distribués au sein de la classe des insectes. Un peptide antibactérien isolé à partir de l'intestin de porc présente une certaine similitude de séquence avec les cécropines d'insectes [7].

Les cécropines comportent entre 35 et 39 résidus ; elles sont dépourvues de cystéines et ne sont pas glycosylées (figure 1). Elles ont typiquement une partie $\mathrm{N}$-terminale fortement basique et un côté C-terminal nettement hydrophobe. Toutes présentent une amidation C-terminale [6]. De nombreux résidus sont conservés en position équivalente (voir Tableau I, p. 435 et article de Nicolas et al., p. 423 de ce numéro). L'analyse de la structure tridimensionnelle par RMN [8] suggère que les cécropines ont deux hélices $\alpha$ : une hélice amphipathique $\mathrm{N}$-terminale (résidus 5 à 21) et une hélice C-terminale (résidus 25 à 37), elle aussi amphipathique mais à un degré moindre. Les deux hélices sont séparées par une région de liaison comportant toujours un résidu glycine et un résidu proline; les axes longs des hélices sont situés dans deux plans qui forment entre eux un angle de 70 à $100^{\circ}$. Les quatre premiers résidus $\mathrm{N}$-terminaux ne sont pas engagés dans le segment hélicoïdal.

Plusieurs gènes codant pour des isoformes de cécropine sont en général présents chez une même espèce. Ainsi, chez la drosophile, trois gènes distincts codant pour trois isoformes sont regroupés dans un cluster de $4 \mathrm{~kb}$ comportant, en plus, deux pseudogènes [9]. Les cécropines sont synthétisées sous forme de précurseurs dont la maturation est réalisée en trois étapes : (1) clivage par une signal peptidase de l'extrémité N-terminale ; (2) coupure par une dipeptidyl aminopeptidase d'un ou de deux dipeptides $\mathrm{N}$-terminaux contenant soit un résidu proline, soit un résidu alanine ; (3) amidation C-terminale [6] Les cécropines ont un large spectre d'activité antibactérienne, aussi bien contre des germes à Gram positif que négatif. En revanche, elles n'agissent pas sur les cellules eucaryotes. Les travaux des équipes de Merrifield (New York) et de Boman (Stockholm), faisant appel à de nombreuses molécules de synthèse (dont des D-énantiomères), conduisent à penser 


\section{RÉFÉRENCES}

1. Cuénot L. Études physiologiques sur les orthoptères. Arch Biol Liège 1896; 14 : 293-341.

2. Paillot A. L'immunité acquise chez les insectes. CR Soc Biol 1920 ; 83 : 278-80.

3. Metalnikow S. Immunité naturelle ou acquise des chenilles de Galleria mellonella. $C R$ Soc Biol Paris 1920 ; 83 : 817-20.

4. Morhig W, Messner B. Immunoreaktionen bei Insekten. I. Lysozym als grundlegender antibakterieller Factor in humoralen Abwehrmechanismus der Insekten. Biol Zentralblatt $1968 ; 87: 439-69$.

5. Steiner $H$, Hultmark D, Engström A, Bennich H, Boman HG. Sequence and specificity of two antibacterial proteins involved in insect immunity. Nature 1981; 292 246-8.

6. Boman HG, Faye I, Gudmundsson GH Lee JY, Lidholm DA. Cell-free immunity in Cecropia. A model system for antibacterial proteins. Eur $J$ Biochem 1991; 201: $23-31$.

7. Lee JY, Boman A, Chuanxin S, et al. Antibacterial peptides from pig intestine isolation of a mammalian cecropin. Proc Natl Acad Sci USA 1989 ; 86 : 9159-62.

8. Holak TA, Engström A, Kraulis PJ, et al. The solution conformation of the antibacterial peptide cecropin A : a nuclear magnetic resonance and dynamical simulated annealing study. Biochemisty $1988 ; 27$ : 7620-9.

9. Kylsten P, Samakovlis C, Hultmark D. The cecropin locus in Drosophila. A compact gene cluster involved in the response to infection. $E M B O J 1990 ; 9$ : 217-24.

10. Wade D, Boman A, Wahlin B, et al. All-D amino acid-containing channelforming antibiotic peptides. Proc Natl Acad Sci USA 1990 ; 87 : 4761-5.

11. Lambert J, Keppi E, Dimarcq JL, $d$ al. Insect immunity : isolation from immune blood of the dipteran Phormia terranovae of two insect antibacterial peptides with sequence homology to rabbit lung macrophage bactericidal peptides. Proc Natl Acad Sci USA 1989 ; 86 : 262-6.

12. Selsted ME, Harwig SLS, Ganz T, Schilling JW, Lehrer RI. Primary structures of three human neutrophil defensins. $J$ Clin Invest $1985 ; 76: 1436-9$.

13. Lehrer RI, Ganz T, Selsted ME Defensins. Endogenous antibiotic peptides of que les cécropines, comme les magainines de peau de xénope et la melittine du venin d'abeille, qui forment également des hélices amphipathiques, agissent sur les membranes bactériennes en créant des canaux ioniques transmembranaires (voir l'article de Nicolas et al., p. 423 de ce numéro). La formation de ces canaux est indépendante d'interactions spécifiques avec des récepteurs chiraux ou des enzymes [10].

\section{- Les défensines d'insectes}

Les défensines d'insectes sont des peptides cationiques, non glycosylés, comprenant 39 à 43 résidus, dont six cystéines. Le nom de défensine d'insecte fut initialement proposé par notre laboratoire pour un peptide de $4 \mathrm{kDa}$ isolé à partir du sang " immun " de larves du diptère Phormia terranovae [11]. La séquence peptidique révélait en effet une certaine similitude avec un groupe de peptides bactéricides présents dans les phagocytes de mammifères et appelés défensines par Lehrer et al. (Los Angeles) en 1985 ([12], revue dans [13]). Nous connaissons actuellement les séquences de 8 défensines d'insectes présentes chez les diptères, hyménoptères et coléoptères. Ces molécules sont apparemment absentes chez les lépidoptères. Toutes les défensines contiennent un motif caractéristique de six cystéines engagées dans trois ponts disulfure intramoléculaires. Une analyse de la structure tridimensionnelle par RMN de la défensine de Phormia dans l'eau a conduit à proposer l'arrangement spatial illustré par la figure 2 [14] : (1) une boucle $\mathrm{N}$-terminale formée par les résidus 1 à 13 ; (2) une hélice $\alpha$ constituée des résidus 14 à 24, qui présente un caractère amphipathique avec une face hydrophobe et une face chargée positivement ; (3) un feuillet $\beta$ antiparallèle comprenant les résidus 27 à 40. L'hélice $\alpha$ est reliée au feuillet $\beta$ par deux ponts disulfure et le troisième pont disulfure lie la cystéine $\mathrm{N}$ terminale également à une cystéine $\mathrm{du}$ feuillet $\beta$. Il est intéressant de noter qu'un arrangement identique stabilisant l'hélice $\alpha$ par l'intermédiaire de deux ponts disulfure qui la relient à un feuillet $\beta$ (cysteine stabilized helix motif) est trouvé dans des toxines de venins de scorpions (par exemple, la charybdotoxine [15]). En revanche, la structure de la défensine d'insecte diffère de façon marquante de celle des défensines de mammifères qui se présentent sous forme d'un feuillet $\beta$ à trois brins antiparallèles et sont dépourvues d'hélice $\alpha[16$, 17]. Cela nous conduit à rejeter l'idée initiale que les défensines d'insectes et les défensines de mammifères sont des molécules homologues dérivant d'un gène ancestral commun.

Les défensines d'insectes sont synthétisées sous forme de préprodéfensines (comme les défensines de mammifères) qui sont transformées en formes actives par coupure d'un peptide signal et d'une proséquence [18].

Les défensines d'insectes sont surtout actives contre des germes à Gram positif. Leur action est extrêmement rapide sur le germe test Micrococcus luteus. Un contact d'une minute avec $0,5 \mu \mathrm{M}$ de défensine est suffisant pour tuer les germes [11]. Les concentrations endogènes de défensine dépassent $1 \mu \mathrm{M}$ dans les heures qui

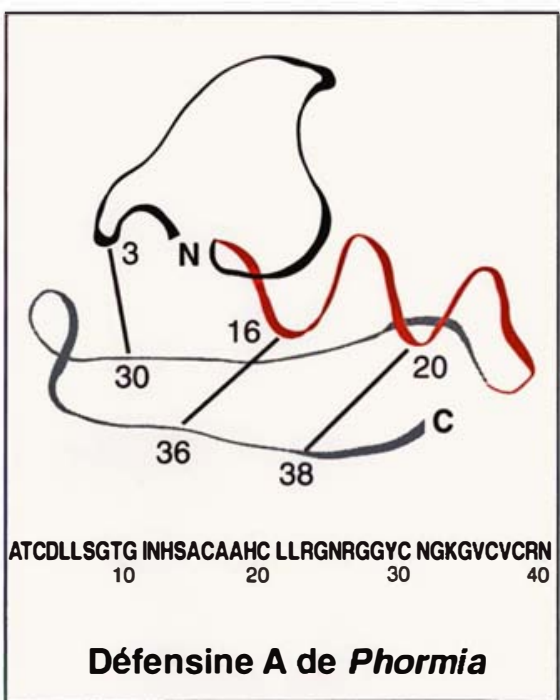

Figure 2. La défensine A du diptère Phormia terranovae: séquence peptidique (code à une lettre) et représentation tridimensionnelle indiquant trois segments distincts : une boucle N-terminale (en noir), une hélice $\alpha$ centrale (en rouge) et un feuillet $\beta$ C-terminal à deux brins antiparallèles. Noter que I'hélice $\alpha$ est reliée au brin $C$-terminal du feuillet $\beta$ par deux ponts disulfure et que la boucle $N$-terminale est reliée par un autre pont disulfure à l'autre brin du feuillet $\beta$. (D'après $[11,14]$, représentation tridimensionnelle inspirée de [14].) 
Tableau 1

SÉQUENCES PEPTIDIQUES DES ATTACINES, SARCOTOXINES, II, COLÉOPTÉRICINE, APIDAECINE, ABAECINE ET DIPTÉRICINE*

\begin{tabular}{|c|c|c|c|c|c|c|c|}
\hline \multicolumn{2}{|c|}{$\begin{array}{l}\text { Attacine basique } \\
\text { Attacine acide }\end{array}$} & $\begin{array}{l}\text { QAGALTINSD } \\
\text { DAHGALTLNS }\end{array}$ & $\begin{array}{l}\text { GTSGAVVKVP } \\
\text { DGTSGAVVKV }\end{array}$ & $\begin{array}{l}\text { ITGNENHKFS } \\
\text { PFAGNDKNIV }\end{array}$ & $\begin{array}{l}\text { ALGSVDLTNO } \\
\text { SAIGSVDLTD }\end{array}$ & $\begin{array}{l}\text { MKLGAATAGL } \\
\text { RQKLGAATAG }\end{array}$ & \\
\hline $\begin{array}{l}\text { AYDNVNGHGA } \\
\text { VALDNINGHG }\end{array}$ & $\begin{array}{l}\text { TLTKTHIPGF } \\
\text { LSLTDTHIPG }\end{array}$ & $\begin{array}{l}\text { GDKMTAAGKV } \\
\text { FGDKMTAAGK }\end{array}$ & $\begin{array}{l}\text { NLFHNDNHDF } \\
\text { VNVFHNDNHD }\end{array}$ & $\begin{array}{l}\text { SAKAFATKNM } \\
\text { ITAKAFATRN }\end{array}$ & $\begin{array}{l}\text { PNIPQVPNFN } \\
\text { MPDIANVPNF }\end{array}$ & $\begin{array}{l}\text { TVGAGVDYMF } \\
\text { NTVGGGIDYM }\end{array}$ & \\
\hline $\begin{array}{l}\text { KDKIGASANA } \\
\text { FKDKIGASAS }\end{array}$ & $\begin{array}{l}\text { AHTDFINRND } \\
\text { AAHTDFINRN }\end{array}$ & $\begin{array}{l}\text { YSLGGKLNLF } \\
\text { DYSLDGKLNL }\end{array}$ & $\begin{array}{l}\text { KTPTTSLDFN } \\
\text { FKTPDTSIDF }\end{array}$ & $\begin{array}{l}\text { AGWKKFDTPF } \\
\text { NAGFKKFDTP }\end{array}$ & $\begin{array}{l}\text { FKSSWEPSTS } \\
\text { FMKSSWEPNF }\end{array}$ & $\begin{array}{l}\text { FSFSKYF } \\
\text { GFSLSKYF }\end{array}$ & \\
\hline \multicolumn{2}{|c|}{$\begin{array}{l}\text { Sarcotoxine } \| 1 A \\
\text { Sarcotoxine } \| 1 \\
\text { Sarcotoxine } \| 2 \\
\text { Sarcotoxine } \| 3\end{array}$} & $\begin{array}{l}\text { QKLPVPIPPP } \\
\text { QKLPVPIPPP } \\
\text { QKLPVPIPPP } \\
\text { QKLPVPIPPP }\end{array}$ & $\begin{array}{l}\text { TNPPVAAFHN } \\
\text { SNPPVAVLQN } \\
\text { TNPPVAAFHN } \\
\text { TNPPVAAFHN }\end{array}$ & $\begin{array}{l}\text { SVATNSKGGQ } \\
\text { SVATNSKGGQ } \\
\text { SVATNSKGGQ } \\
\text { SVATNSKGGQ }\end{array}$ & $\begin{array}{l}\text { DVSVKLAATN } \\
\text { DVSVKLSATN } \\
\text { DVSVKLAATN } \\
\text { DVSVKLAATN }\end{array}$ & $\begin{array}{l}\text { LGNKHVQPIA } \\
\text { LGNNHVQPIA } \\
\text { LGNKHVQPIA } \\
\text { LGNKHVQPIA }\end{array}$ & \\
\hline $\begin{array}{l}\text { EVFAEGNTKG } \\
\text { EVFAEGNTKG } \\
\text { EVFAKGNTQG } \\
\text { EVFAEGNTKG }\end{array}$ & $\begin{array}{l}\text { GNVLRGATVG } \\
\text { GNVLRGATVG } \\
\text { GNVLRGATVG } \\
\text { GNVLRGATVG }\end{array}$ & $\begin{array}{l}\text { VQGHGLGASV } \\
\text { VQGHGLGASV } \\
\text { VQGHGLGASV } \\
\text { VQGHGLGASV }\end{array}$ & $\begin{array}{l}\text { TKSQDGIAES } \\
\text { TKT------. } \\
\text { TKTQDGIAES } \\
\text { TKSGNGIAES }\end{array}$ & $\begin{array}{l}\text { FRKQAEANLR } \\
\text { FRKQAEANLR } \\
\text { FRKQAEANLR }\end{array}$ & $\begin{array}{c}\text { LGDSASLIGK } \\
\text { LGDSASLIGK } \\
\text { LGDSASLIGK }\end{array}$ & $\begin{array}{l}\text { VSQTDTKIKG } \\
---Q T D T K I K G \\
\text { VSQTDTKIKG } \\
\text { VSQTDTKIKG }\end{array}$ & \\
\hline $\begin{array}{l}\text { IDFKPQLSSS } \\
\text { LDFQPQLSSS } \\
\text { IDFKPQLSSS } \\
\text { IDFKPQLSSS }\end{array}$ & $\begin{array}{l}\text { SLALQGDRLG } \\
\text { TLALQGDRLG } \\
\text { SLALQGDRLG } \\
\text { SLALQGDRLG }\end{array}$ & $\begin{array}{l}\text { ASISRDVNRG } \\
\text { ASISRDVNRG } \\
\text { ASISRDVNRG } \\
\text { ASISRDVNRG }\end{array}$ & $\begin{array}{l}\text { VSDTLTKSVS } \\
\text { VSDTFTKSVS } \\
\text { VSDTLTKSIS } \\
\text { VSDTLTKSIS }\end{array}$ & $\begin{array}{l}\text { ANLFRNDNHN } \\
\text { ANVFRNDNHN } \\
\text { ANVFRNDNHN } \\
\text { ANVFRNDNHN }\end{array}$ & $\begin{array}{l}\text { LDASVFRSDV } \\
\text { LDATVFRSDV } \\
\text { LDASVFRSDV } \\
\text { LDASVFRSDV }\end{array}$ & $\begin{array}{l}\text { RQNNGFNFQK } \\
\text { RQNNGFNFQK } \\
\text { RONNGFNFQK } \\
\text { RONNGFNFOK }\end{array}$ & \\
\hline $\begin{array}{l}\text { TGGMLDYSHA } \\
\text { TGGMLDYSHA } \\
\text { TGGMLDYSHA } \\
\text { TGGMLDYSHA }\end{array}$ & $\begin{array}{l}\text { NGHGLNAGLT } \\
\text { NGHGLNAGLT } \\
\text { NGHGLNAGLT } \\
\text { NGHGLNAGLT }\end{array}$ & $\begin{array}{l}\text { RFSGIGNQAT } \\
\text { HFSGIGNQAN } \\
\text { RFSGIGNQAN } \\
\text { RFSGIGNQAN }\end{array}$ & $\begin{array}{l}\text { VGGYSTLFRS } \\
\text { VGGSSTLFKS } \\
\text { VGGYSTLFRS } \\
\text { VGGYSTLFRS }\end{array}$ & $\begin{array}{l}\text { NDGLTSLKAN } \\
\text { NDGSLSLKAN } \\
\text { NDGLTSLKAN } \\
\text { NDGLTSLKAN }\end{array}$ & $\begin{array}{l}\text { AGGSQWLSGP } \\
\text { AGGSOWLSGP } \\
\text { AGGSOWLSGP } \\
\text { AGGSOWLSGP }\end{array}$ & $\begin{array}{l}\text { FANQRDYSFG } \\
\text { FSNQRDYNVG } \\
\text { FANQRDYSFG } \\
\text { FANQRDYSFG }\end{array}$ & $\begin{array}{l}\text { LGLSHNAWR* } \\
\text { LSLTHHGCG* } \\
\text { LGLSHNAWR* } \\
\text { LGLSHNAWR* }\end{array}$ \\
\hline $\begin{array}{r}\text { Coléc } \\
\text { GWGKVIRGPN }\end{array}$ & $\begin{array}{l}\text { téricine } \\
\text { KAKPTWHVGG }\end{array}$ & $\begin{array}{l}\text { SLQGGAPNFP } \\
\text { TYRR }\end{array}$ & OPSOQNGGWO & VSPDLGRDDK & GNTRGQIEIQ & NKGKDHDFNA & \\
\hline $\begin{array}{r}A p i \\
A b\end{array}$ & $\begin{array}{l}\text { aecine } \\
\text { ecine }\end{array}$ & $\begin{array}{l}\text { GNNRPVYIPQ } \\
\text { YVPLPNVPQP }\end{array}$ & $\begin{array}{l}\text { PRPPHPRI } \\
\text { GRRPFPTFPG }\end{array}$ & QGPFNPKIKW & PQGY & & \\
\hline $\begin{array}{l}\text { Diptérici } \\
\text { Diptéricin }\end{array}$ & $\begin{array}{l}\text { Phormia } \\
\text { Drosophila }\end{array}$ & $\begin{array}{l}\text { DEKPKLILPT } \\
\text { DDMTMKPTPP }\end{array}$ & $\begin{array}{l}\text { PAPPNLPQLV } \\
\text { PQYPLNLQGG }\end{array}$ & $\begin{array}{l}\text { GGGGGNRKDG } \\
\text { GGGQSGDGFG }\end{array}$ & $\begin{array}{l}\text { FGVSVDAHQK } \\
\text { FAVQGHQKVW }\end{array}$ & $\begin{array}{l}\text { VWTSDNGRHS } \\
\text { TSDNGRHEIG }\end{array}$ & \\
\hline $\begin{array}{l}\text { IGVTPGYSQH } \\
\text { LNGGYGQHLG }\end{array}$ & $\begin{array}{l}\text { LGGPYGNSRP } \\
\text { GPYGNSEPSW }\end{array}$ & $\begin{array}{l}\text { DYRIGAGYSY } \\
\text { KVGSTYTYRF }\end{array}$ & $\begin{array}{l}\text { NFG } \\
\text { PNF }\end{array}$ & & & & \\
\hline
\end{tabular}

* Voir le texte pour commentaires et références.

suivent l'induction de la réponse antibactérienne. Le mode d'action n'est pas connu avec précision ; la molécule a une forte affinité pour la cardiolipine, un phospholipide membranaire, et cette affinité semble essentielle pour l'activité [19].

- Les peptides riches en résidus proline : les apidaecines, l'abaecine et les drosocines (Tableau I)

Dans une étude récente sur le sang "immun" d'abeilles, le groupe de Casteels, à Gand, a mis en évidence la présence de petits peptides riches en proline $(30 \%)$, dépourvus de cystéine et essentiellement actifs contre les germes à Gram négatif [20, 21]. Ces molécules, qui semblent être les peptides antibactériens majeurs de la réponse immunitaire de l'abeille, appartiennent à deux types : (1) les apidaecines (de Apis, abeille) [20], dont plusieurs isoformes ont été caractérisées et qui comportent chacune 18 résidus; (2) l'abaecine [21] constituée de 34 résidus et qui présente dans sa partie $\mathrm{N}$-terminale une homologie de séquence avec les apidaecines, ainsi qu'avec le domaine riche en proline des diptéricines (voir ci-dessous). La présence des peptides antibactériens riches en proline est certainement plus répandue chez les insectes qu'il n'a été admis jusqu'ici. Nous venons d'isoler au laboratoire de petits peptides riches en proline chez des drosophiles "immunisées" où elles représentent également des molécules majeures de la réponse antibactérienne dirigée contre les germes à Gram négatif. Ces molécules, que nous appelons drosocines, feront l'objet d'une description détaillée ultérieurement.

- Les polypeptides antibactériens inductibles : les attacines, les sarcotoxines II, les diptéricines et la coléoptéricine (Tableau I, figure 3, p. 437).

Nous regroupons ici plusieurs polypeptides dont les masses sont supérieures à $8 \mathrm{kDa}$ et peuvent atteindre $27 \mathrm{kDa}$ (sarcotoxines II). Tous sont dépourvus de cystéines et sont actifs contre les germes à Gram négatif. A l'exception des attacines, tous sont riches en glycine. Les diptéricines présentent deux domaines distincts : 


\section{RÉFÉRENCES}

14. Bonmatin JM, Bonnat JL, Gallet X, et al. Two-dimensional ${ }^{1} \mathrm{H}-\mathrm{NMR}$ study of recombinant insect defensin $A$ in water. Resonance assignments, secondary structure and global folding. J Biomol NMR 1992 (sous presse).

15. Bontems F, Roumestand C, Gilquin B, Ménez A, Toma $F$. Refined structure of charybdotoxin : common motifs in scorpion toxins and insect defensins. Science 1991 . $254: 1521-3$.

16. Pardi A, Hare DR, Selsted ME, Morrison RD, Bassolino DA, Bach AC. Solution structures of the rabbit neutrophil defensin NP-5. J Mol Biol 1988; 201 : 625-36.

17. Hill CP, Yee J, Selsted ME, Eisenberg D. Crystal structure of defensin HNP-3, an amphiphilic dimer : mechanisms of membrane permeabilization. Science 1991; 251 : 1481-5.

18. Dimarcq JL, Zachary D, Hoffmann JA, Hof fmann D, Reichhart JM. Insect immunity : expression of the two major inducible antibacterial peptides, defensin and diptericin, in Phormia tertanovae. EMBO J 1990 $9:$ 2507-15.

19. Matsuyama K, Natori S. Mode of action of sapecin, a novel antibacterial protein of Sarcophaga peregrina (llesh fly). J Biochem $1990 ; 108: 128-32$.

20. Casteels P, Ampe C, Jacobs F, Vaeck M, Tempst P. Apidaecins : antibacterial peptides from honey bees. EMBO J $1989 ; 8$ : 2387-91.

21. Casteels $P$, Ampe $C$, Rivière L, et al. Isolation and characterization of abaecin, major antibacterial response peptide in the honey bee (Apis mellifera). Eur $J$ Biochem 1990 ; 187 : 381-6

22. Kockum K, Faye I, von Hofsten $P$ Lee Y, Xanthopoulos KG, Boman HG Insect immunity. Isolation and sequence of two cDNA clones corresponding to acidic and basic attacins from Hyalophora cecropia. $E M B O J 1984 ; 3$ : 2071-5.

23. Ando K, Natori S. Molecular cloning, sequencing and characterization of $\mathrm{cDNA}$ for sarcotoxin IIA, an inducible antibacterial protein of Sarcophaga peregrina (flesh fly). Biochemistry $1988 ; 27$ : 1715-21.

24. Dimarcq JL, Keppi E, Dunbar B, et al Insect immunity. Purification and characterization of a family of novel inducible antibacterial proteins from immunized larvae of the dipteran Phormia terranovae and complete amino acid sequence of the predominan member, diptericin A. Eur J Biochem 1988 . $171: 17-22$. le domaine $\mathrm{N}$-terminal est riche en proline et apparenté aux apidaecines, et le domaine C-terminal, riche en glycine, présente une similitude de séquence avec deux domaines trouvés dans les attacines et les sarcotoxines II. Nous discuterons ci-dessous l'hypothèse qu'il existe une relation phylogénétique entre ces polypeptides. La coléoptéricine est riche en glycine mais ne présente pas de similitude de séquence avec les autres polypeptides antibactériens connus.

Les attacines sont des polypeptides bactériostatiques inductibles de taille supérieure à $20 \mathrm{kDa}$, découverts par l'équipe de Boman (Stockholm) dans le sang "immun" de Hyalophora cecropia (lépidoptère de la famille des Attacidae, d'où le nom proposé). Six attacines, comportant entre 184 et 188 résidus, ont été isolées ; quatre sont de nature basique et deux sont neutres ou légèrement acides. L'homologie de séquence entre les attacines basiques et acides est voisine de $80 \%$ [22]. Les attacines ont deux domaines C-terminaux de 60 acides aminés comportant une similitude de séquence avec les domaines Cterminaux des sarcotoxines II (domaines $\mathrm{G} 1$ et $\mathrm{G} 2$ ) et des diptéricines (domaine $\mathrm{G}$, voir figure 3 et discussion ci-dessous).

Le génome de $H$. cecropia contient un locus attacine renfermant un seul gène $d$ 'attacine basique et un seul gène d'attacine acide, ainsi que deux pseudogènes. Les deux gènes actifs comportent chacun un intron en position homologue et ils sont transcrits en sens inverse. Les produits des deux gènes, les préproattacines, sont transformés en produits mûrs par coupure d'un peptide signal et d'une proséquence : les six isoformes résultent ensuite d'une coupure partielle du tétrapeptide sérine-lysine-tyrosine phénylalanine C-terminal et, dans le cas des attacines basiques, d'une cyclisation partielle de la glutamine $\mathrm{N}$-terminale. Ainsi les modifications post-traductionnelles des produits de deux gènes expliquent l'apparition concomitante de six isoformes d'attacines [6].

Chez le diptère Sarcophaga peregrina, de grands polypeptides antibactériens inductibles riches en glycine ont été mis en évidence par le groupe de Natori à Tokyo. Il s'agit d'une famille de plusieurs membres (au moins six), appelés sarcotoxines II (de Sarcophaga et toxine antibactérienne) [23]. Ces molécules sont actives contre des germes à Gram négatif et semblent inhiber la synthèse de la paroi cellulaire. La première molécule caractérisée, la sarcotoxine IIA, comporte 270 résidus; comme les autres membres de la famille, elle présente un pyroglutamate $\mathrm{N}$-terminal et est amidée du côté C-terminal. Les sarcotoxines II sont dépourvues de cystéine mais riches en glycine : les résidus glycine sont. regroupés en deux domaines d'une soixantaine de résidus du côté C-terminal, qui présentent une certaine similitude avec les domaines C-terminaux des attacines (domaines $\mathrm{G} 1$ et $\mathrm{G} 2$, figure 3).

Les diptéricines forment une famille de peptides de 82 ou 83 résidus isolés au laboratoire et décrits pour l'instant seulement chez deux diptères, Phormia terranovae et Drosophila melanogaster [24, 25]. Ce sont des molécules cationiques présentant un court domaine $\mathrm{N}$-terminal riche en résidus proline et un long domaine central et C-terminal de 60 résidus très riche en glycine. Ces molécules sont synthétisées sous forme de prédiptéricines comportant un peptide signal et la séquence diptéricine. La diptéricine de Phormia serait amidée au niveau du résidu C-terminal [26] ; contrairement à celle de la drosophile [25]. Une caractéristique remarquable de toutes les diptéricines est la présence de cinq résidus glycine successifs à la limite des deux domaines proline et glycine, ce qui doit conférer une extrême flexibilité à cette région de la chaîne polypeptidique.

Le gène de la diptéricine a été récemment caractérisé chez la drosophile [27]. Il est dépourvu d'intron et code pour une prédiptéricine à partir de laquelle est formée la diptéricine par l'action d'une signal peptidase et d'une dipeptidyl aminopeptidase.

Les diptéricines sont principalement actives contre les germes à Gram négatif en agissant sur la membrane cytoplasmique des bactéries.

L'analyse détaillée des séquences des diptéricines, des attacines et des sarcotoxines II suggère que ces familles de polypeptides antibactériens sont phylogénétiquement reliées [25]. Les attacines et sarcotoxines II ont deux 


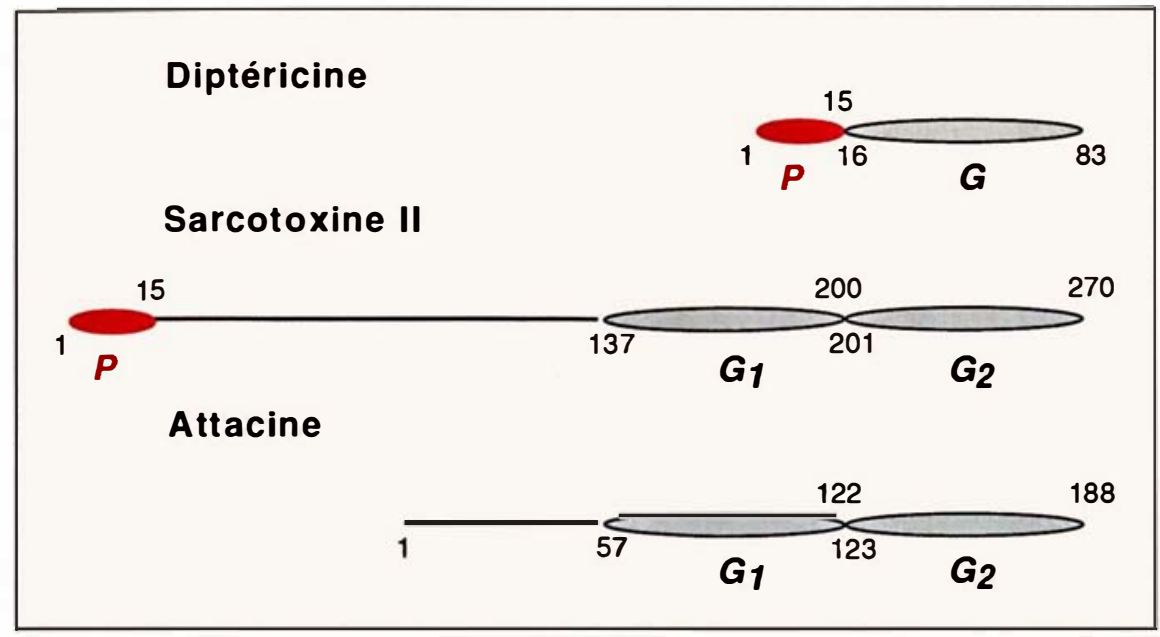

Figure 3. Représentation des domaines riches en proline (P) et glycine (G) de la diptéricine et comparaison avec l'organisation en domaines présentant des similitudes de séquence respectivement dans les sarcotoxines II et les attacines. Les chiffres réfèrent aux résidus de la séquence peptidique. Les domaines $P$ sont courts et les domaines $G$ comportent une soixantaine de résidus dans les trois familles représentées sur la figure. Voir le texte pour la discussion d'une éventuelle relation phylogénétique entre ces polypeptides. (D'après [25].)

domaines d'une soixantaine de résidus relativement riches en glycine (domaines G1 et G2) du côté C-terminal, qui présentent des similitudes entre eux et avec le domaine $G$ de la diptéricine. Les similitudes de séquence sont, par exemple, de $34 \%$ entre le domaine G2 de la sarcotoxine IIA et le domaine $\mathrm{G}$ de la diptéricine ; en général, elles sont supérieures à $20 \%$ entre les différents domaines $\mathrm{G}$ et de nombreuses différences sont dues à des remplacements conservateurs. Il est intéressant de souligner que dans le cas des attacines, chacun des deux domaines $G$ est codé par un exon séparé. Par ailleurs, les sarcotoxines II comportent, comme les diptéricines, un domaine riche en proline (domaine P) du côté N-terminal. Rappelons que le domaine $\mathrm{P}$ des diptéricines présente des similitudes de séquence avec les apidaecines. La conservation de courts domaines $\mathrm{P}$ et de longs domaines $G$ suggère que ces domaines correspondent à des unités fonctionnelles. Les diptéricines, en associant un domaine $\mathrm{P}$ et un seul domaine $G$, pourraient ainsi définir une structure minimale d'un membre d'une nouvelle superfamille de polypeptides bactéricides.

La coléoptéricine a été isolée au $\mathrm{m} / \mathrm{s} n^{\circ} 5$, vol. 8, mai 92
L'analyse de profils transcriptionnels de différents gènes codant pour des peptides antibactériens au cours de la réponse immunitaire et les travaux d'hybridation in situ, aboutit aux conclusions suivantes qui confirment et étendent celles présentées plus haut : (1) chez les insectes normaux, les gènes des peptides antibactériens sont en général silencieux; (2) l'induction est un phénomène rapide qui peut être mis en évidence dès 30 minutes après injection bactérienne ; (3) l'expression des gènes est un phénomène transitoire et s'arrête, selon les espèces étudiées, au bout de 2 à 3 jours ; (4) une nouvelle stimulation réalisée chez des insectes dont la réponse à une première injection s'est éteinte, déclenche une réponse identique à la première : l'insecte ne garde pas de mémoire de la première injection; (5) les cellules du corps gras, ainsi que certains types de cellules sanguines, répondent à la stimulation par la transcription concomitante des gènes codant pour plusieurs types de peptides antibactériens ; (6) cette transcription peut être induite par divers types de stimuli.

Ces observations conduisent à poser la question du contrôle de l'expression. Plusieurs équipes abordent actuellement l'analyse du contrôle de l'expression des gènes codant pour les peptides antibactériens chez la drosophile, qui constitue un modèle biologique éminemment favorable pour ce type d'études. Une approche expérimentale actuellement mise à profit dans l'étude des réactions antibactériennes est l'établissement de mouches transgéniques par l'intermédiaire du transposon P. Nous avons illustré la démarche par la figure 4, page 438, qui présente une mouche transgénique porteuse d'un gène chimère " promoteur de diptéricine/rapporteur $\beta$ galactosidase " [27]. En bref, un des peptides antibactériens inductibles de la drosophile appartient à la famille des diptéricines [25]. Le gène codant pour ce peptide a été cloné au laboratoire et une construction a été réalisée dans laquelle les séquences potentiellement régulatrices situées dans la partie amont du gène de la diptéricine ont été fusionnées à la séquence codante d'un gène rapporteur lac $Z$. Lorsque le gène chimère est exprimé, son produit protéique, la 


\section{RÉFÉRENCES}

25. Wicker C, Reichhart JM, Hoffmann D, Hultmark D, Samakovlis C, Hoffmann JA. Characterization of a Drosophila cDNA encoding a novel member of the diptericin family of immune peptides. J Biol Chem $1990 ; 265$ : 22493-98.

26. Reichhart JM, Essrich M, Dimarcq JL, Hoffmann D, Hoffmann JA, Lagueux M. Insect immunity. Isolation of $\mathrm{cDNA}$ clones corresponding to diptericin, an inducible antibacterial peptide from Phormia terranovae (Diptera). Transcriptional profiles during immunization. Eur $J$ Biochem 1989 ; 182 : 423-7.

27. Reichhart JM, Meister M, Dimarcq JL, et al. Insect immunity : developmental and inducible activity of the Drosophila diptericin promoter. EMBOJ 1992 ; 11 (sous presse).

28. Bulet $\mathrm{P}$, Cociancich S, Dimarcq JL, et al. Isolation from a coleopteran insect of a novel inducible antibacterial peptide and of new members of the insect defensin family. J Biol Chem 1991; 266 : 24520-5.

29. Isshiki H, Akira S, Sugita $T$, et al. Reciprocal expression of NF-IL6 and C/EBP in hepatocytes : possible involvement of NF-IL6 in acute phase protein gene expression. New Biol 1991; 3 : 63-70

30. Baeuerle PA. The inducible transcription activator $\mathrm{NF}-\kappa \mathrm{B}$ : regulation by distinct protein subunits. Biochim Biophys Acto 1991 ; 1072 : 63-80.

31. Urban MB, Schreck R, Baeuerle PA. NF $-\kappa \mathrm{B}$ contacts DNA by a heterodimer of the p50 and p65 subunit. EMBO J 1991 ; $10: 1817-25$

32. Jomori T, Natori S. Molecular cloning of cDNA for lipopolysaccharide-binding protein from the hemolymph of the American cockroach, Periplaneta americana. J Biol Chem 1991 ; 266 : 13318-23.

33. Takahashi $\mathrm{H}$, Komano $\mathrm{H}$, Natori $\mathrm{S}$. Expression of the lectin gene in Sarcophaga peregrina during normal development and under conditions where the defence mechanism is activated. $J$ Insect Physiol $1986 ; 32$ 771-9.

34. Sun SC, Lindström I, Boman HG, Faye I, Schmidt O. Hemolin : an insect immune protein belonging to the immunoglobulin superfamily. Science 1990 ; 250 : 1729-32.

35. Ladenforff NE, Kanost MR. Bacteriainduced protein P4 (hemolin) from Manduca sexta : a member of the immunoglobulin superfamily which can inhibit hemocyte aggregation. Arch Insect Biochem Physiol 1991 ; $18: 285-300$

36. Janeway CA. Approaching the asymptote ? Evolution and revolution in immunology. Cold Spring Harbor Symposia on Quan- $\beta$-galactosidase, transforme le substrat incolore X-gal en une substance bleue facilement visualisée. Cette construction a été introduite dans un vecteur approprié comportant des éléments du transposon $\mathrm{P}$; après injection dans la zone des cellules germinales, localisée au pôle postérieur d'embryons de drosophile, des lignées de mouches ont été établies dans lesquelles le transgène est inséré de façon stable au niveau chromosomique. La mouche transgénique présentée sur la figure 4 a subi l'injection de bactéries 4 heures avant d'être sacrifiée et en présence du substrat X-gal : on voit que toutes les cellules du corps gras, et ces cellules seulement, sont bleues. Des témoins non stimulés restent incolores. Ce résultat indique que l'expression du transgène est, comme le gène de la diptéricine, inductible par injection de bactéries et restreinte aux cellules du corps gras. Nous pouvons conclure que les éléments régulateurs nécessaires à ce type d'expression sont présents dans les séquences placées en amont du gène rapporteur [27]. Une analyse plus fine, utilisant des séquences régulatrices plus courtes ou modifiées contrôlant l'expression du même gène rapporteur, a permis de réaliser une dissection fonctionnelle du promoteur du gène de la diptéricine. Soulignons la présence dans ce promoteur, de plusieurs motifs nucléotidiques identiques ou très proches de motifs consensus de fixation d'activateurs transcriptionnels présents dans les promoteurs de gènes codant pour des protéines de la réponse de phase aiguë exprimés dans le foie de mammiferes [27].

Trois types de motifs paraissent particulièrement intéressants dans ce contexte : (1) le motif de liaison de NFIL-6. Le promoteur du gène de la diptéricine renferme plusieurs copies du motif consensus pour le site de liaison de l'activateur nucléaire qui confère, dans le foie, l'inductibilité par l'interleukine 6 à des gènes codant pour des protéines de phase aiguë [29] ; (2) un motif de liaison identique à la séquence consensus de liaison de NF- $\kappa$ B. Le facteur nucléaire

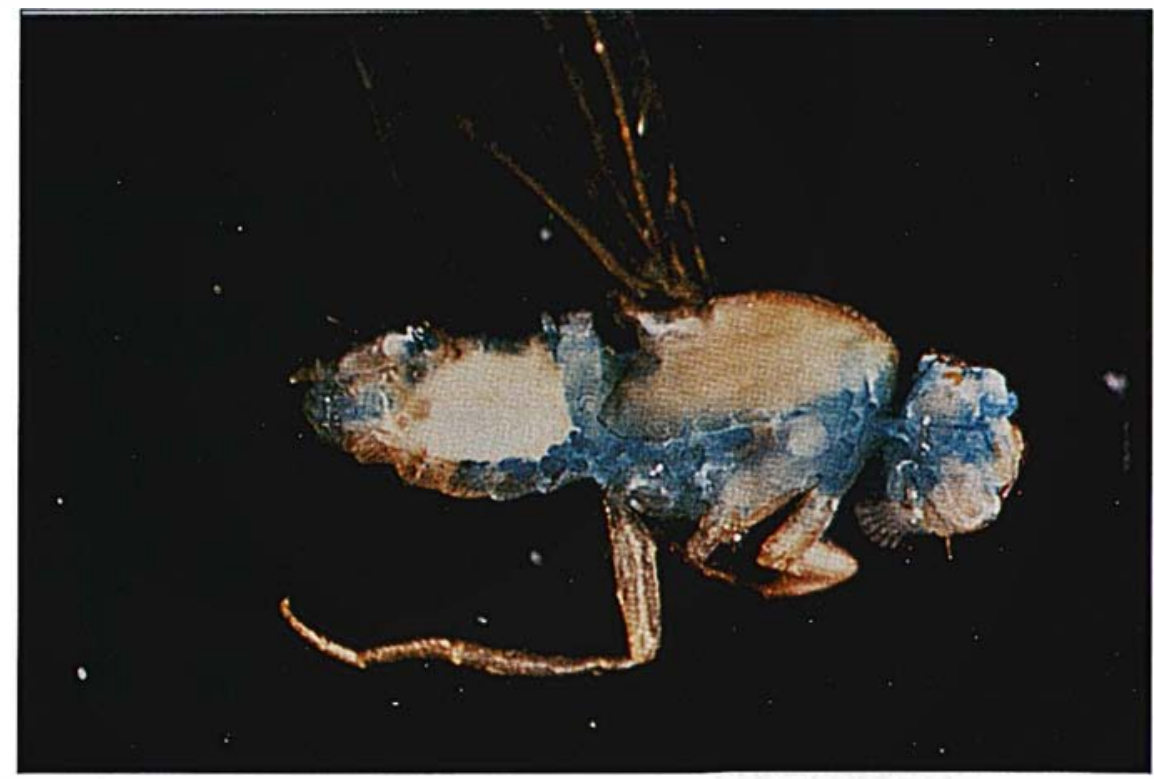

Figure 4. Adulte de drosophile appartenant à une lignée transgénique ayant intégré un gène chimère promoteur de diptéricine/rapporteur $\beta$ galactosidase. Cette mouche a subi une injection d'une faible dose de bactéries et a été disséquée quatre heures après en présence du substrat $X$-gal. La $\beta$-galactosidase exprimée sous le contrôle du promoteur de la diptéricine a transformé ce substrat incolore en un produit bleu. On note que seules les cellules du corps gras ont répondu à la stimulation. Une mouche transgénique témoin qui n'a pas été stimulée par injection de bactéries n'exprime pas le transgène et reste incolore après dissection en présence du substrat $X$-gal (résultat non illustré). (D'après [27].) 
$\kappa \mathrm{B}$ induit la transcription d'une grande variété de gènes en se liant à des motifs nucléotidiques décamériques. La plupart des gènes cibles de cet activateur transcriptionnel sont des cytokines immunomodulatrices, des récepteurs membranaires impliqués dans la réponse immunitaire ou des protéines de phase aiguë $[30,31]$; (3) deux motifs heptadécamériques identiques comportant en leur centre une séquence décamérique très proche (à un nucléotide près) du motif consensus NF- $\kappa \mathrm{B}$. Ce motif décamérique $\mathrm{NF}-\kappa \mathrm{B}-l i k e$ est présent dans plusieurs autres gènes codant pour des peptides antibactériens inductibles d'insectes : cécropine, attacine, lysozyme de $\mathrm{Hya}$ lophora cecropia [6], cécropine de drosophile [9]. L'analyse fonctionnelle de ces motifs est en cours, notamment par transgenèse ; les premiers résultats montrent la nécessité des sites heptadécamériques pour l'induction du gène de la diptéricine.

\section{Reconnaissance de l'agression bactérienne}

Chez les insectes, la reconnaissance de l'agression bactérienne semble relever de la détection de motifs structuraux essentiellement représentés dans les parois bactériennes et rarement ou jamais présents dans les membranes cellulaires des eucaryotes supérieurs, comme les lipopolysaccharides et les peptidoglycanes. Cette reconnaissance est le fait de récepteurs cellulaires et circulants. Des protéines liant les lipopolysaccharides et les peptidoglycanes, ainsi que des lectines fixant des sucres de la paroi bactérienne, ont été isolées dans le sang d'insectes $[32,33]^{*}$. Il apparaît que les protéines de reconnaissance des insectes fixant des motifs structuraux procaryotiques correspondent aux pattern recognition receptors (récepteurs de reconnaissance des motifs) définis par Janeway [36]. Il s'agit d'un type non clonal de défense de l'hôte qui représente la forme primitive de la réponse immunitaire. A celle-ci, les vertébrés, et apparemment eux seuls, ont ajouté la reconnaissance

\footnotetext{
* Chez les lépidoptères, on a récemment caractérisé des protéines qui se fixent sur E. Coli et qui présentent une structure peptidique qui les apparente à la superfamille des immunoglobulines. Il n'y a pas d'indication en faveur d'un réarrangement somatique des gènes codant pour ces molécules, appelées hémolines [34,35].
}

$\mathrm{m} / \mathrm{s} n^{\circ} 5$, vol. 8, mai 92 des antigènes par des récepteurs distribués de façon clonale et codés par des segments de gènes subissant des réarrangements somatiques. Le système de discrimination des insectes entre le non-soi inf ectieux et le sol non infectieux ne permet pas le développement d'une mémoire immunologique. Ainsi, les expériences pionnières de Metalnikow [3] sur l'induction chez des chenilles, d'une protection contre des doses létales de bactéries, par injection préalable d'une faible dose, doivent être interprétées dans un contexte différent de celui d'une immunisation chez les mammifères. La première injection induit, comme nous l'avons vu, l'apparition dans le sang de plusieurs familles de peptides antibactériens qui confèrent de facto une protection contre l'injection subséquente de doses élevées de bactéries. Mais la réponse est limitée dans le temps, et lorsqu'elle s'est éteinte, une nouvelle injection de bactéries induit une réponse identique à la première, dont l'insecte n'a apparemment conservé aucune mémoire.

\section{Conclusions}

Les insectes supérieurs combattent les infections par un mécanisme de réponse très efficace qui implique la synthèse et la sécrétion dans le sang de substances antibactériennes multiples à large spectre d'activité. La réponse est activée par des récepteurs à distribution non clonale qui reconnaissent des motifs structuraux largement distribués dans les enveloppes des procaryotes, mais qui sont absents ou faiblement représentés chez les eucaryotes.

La réponse antibactérienne des insectes évoque par de nombreux aspects la réponse de phase aiguë des mammifères : rapidité de l'induction, nonspécificité, caractère non adaptatif. Il est frappant de voir que les sites principaux de cette réponse sont, chez l'insecte, le corps gras et, chez les mammiferes, le foie : ces deux organes présentent en effet de nombreuses autres analogies fonctionnelles. Le parallélisme entre la réponse antibactérienne des insectes et la réponse de phase aiguë des mammifères est également illustré par la présence, dans les promoteurs des gènes impliqués dans ces deux réponses, de séquences consensus identiques sur lesquelles se fixent des activateurs transcriptionnels intervenant dans le contrôle de leur expression

\section{Summary \\ Inducible antibacterial peptides of insects}

Insects belonging to the phylogenetically recent orders of Lepidoptera, Diptera, Hymenoptera and Coleoptera possess a remarkably efficient antibacterial defense mechanism involving the synthesis of several potent antibacterial peptide families with a large spectrum of activity. The best known of these molecules are the cecropins (4 kDa) which form two amphipathic helices and the insect defensins (4 kDa) characterized by an amphipathic $\alpha$-helix linked via two disulfide bridges to an antiparallel $\beta$-sheet (cysteine stabilized $\alpha$-helix motif). The peptide sequences of seven other inducible antibacterial peptide families have been established over the last years. These are predominantly small cationic proline rich peptides $(2-3 \mathrm{kDa})$ or larger glycine rich polypeptides (8-27 kDa). The synthesis of the antibacterial peptides takes place in the fat body, a functional equivalent of the vertebrate liver. The antibacterial response is activated by non-clonally distributed receptors recognizing structural motifs primarily present on procaryotic cell envelopes (lipopolysaccharides, peptidoglycans). The response is rapid (several hours) and shortlived (several days). There is no indication at present for the existence of an immune memory in these insect orders. The antibacterial response of insects shares many of the characteristics of the acute phase response in mammals.

\section{TIRÉS A PART}

\section{J. A. Hoffmann}

